\title{
The Application of Interesting Graphic Elements in the Poster Design.
}

\author{
Ling $\mathrm{Li}^{1, \mathrm{a}}$ \\ ${ }^{1}$ Zhengzhou University of Industrial Technology, Zhengzhou, Henan, 451100 \\ a email
}

\section{Keywords: Poster Design, Fun Graphics, Graphic Elements, Application}

\begin{abstract}
With the development of society, more and more abundant forms of products, from the original demand of the market converted into oversupply, the choice of a variety of ways, require manufacturers to advertise in order to attract buyers. Poster design occupies an important position in the ad. Into the poster design fun graphic elements enables the entire ad design is full of fun, grab the attention of consumers. Fun poster graphic elements are important design technique of expression. Fun graphics integration requires poster design, showing all the design concept. Enhance the fun poster design, practicality, simplicity. Developments and trends in this paper poster design, the importance of poster design fun graphic elements starting to analyze.
\end{abstract}

\section{Introduction}

With the development of society, the information age now become an important focus of development, poster design also entered a historical dimension. Pattern as an important design element, when the poster design, with its large volume of information, ornamental strong, and high artistic content, extensive use and poster design. Inseparable convey visual graphic design poster graphic design as inside a special and important form of design, integration into the volume of information, the expression of the idea of carrying things, wherein the carrier is a graphical representation of the colors, text these three elements. Fun integrate graphic elements occupy an important role, it is directly related to the success of the entire poster design.

Fun is an important design element graphic references into positive poster design, and now this trend is the development of society can not do without. It is a pluralistic society, the concept of design to highlight the need to create something new out of the ordinary, to meet the needs of the public, to win the public's eye.

\section{The Development Status of Poster Design}

Poster design technique of expression is now an important design element in an artistic, convey a new visual experience people, graphics, color, texture and other aspects of visual elements are fused show poster design artistic charm. Our development has a history of poster design period, the comprehensive concept of traditional culture, show that out of the meaning of culture, but also with other countries is not the same.

China started the development of poster art in comparison with Western countries started a bit late, but quickly highlight the superiority of socialist culture in the tide. Distinctive national characteristics of the 1980s and 1990s poster work performance, and this time is inseparable from the social environment, some of the poster designers in learning the West, Japan and China Taiwan foundation of modern poster design, combined with China's traditional building belonging to China poster art of design. The most prominent manifestations after the Opium War, combined with China's actual merchant, expand the product of their own propaganda, such as the 1980s Wang Hanqing design "fish card padlock," Shao Lichen designed "tube Pa" and so on. These are the times when the integration of the background, poster design and integration interesting graphic elements, won the crowd's attention, visual sense to achieve a fresh, conducive to the promotion poster design concept.

With economic development, the increasingly fierce competition in goods, the contemporary poster design philosophy began to favor vulgarization, leading cultural connotation poster design 
becomes low. Specific performance in two aspects: one is the concept of consumption of unhealthy commodities supplier mislead consumers, encouraging consumers to establish healthy consumption concept, so that consumers blind comparisons with each other, resulting in a waste of resources market; the other hand is using celebrity, to promote its goods exaggerate the effect of the publicity and the actual effect is not consistent, poster design characters referenced large scale, resulting in a vulgar poster design. For this type of phenomenon, public and personal needs come up with specific measures to avoid this kind of social phenomenon, if required net Posters vulgarization phenomenon.

\section{Interesting Graphic Elements Occupy an Important Position in the Poster Design}

Poster design, there are three important factors: graphics, text, color. Graphics is particularly important in poster design, occupies an important position. In the field of graphic design poster is one of the oldest performance art design techniques. Until today, the bill is still recognized as the graphic art and design is the most important design.

The most intuitive visual expression of human emotion is a graphical symbol. Graphical elements from the beginning of the ancient notes will occupy an important position, the graphics are an important means of human memory storage and dissemination of knowledge, but also the direct exchange of information and an effective means of rapid communication.

Pattern as a poster in the major design elements, poster design is unique, has a special significance visual language, is an important performance poster design expression. Graphics more than text and color performance, it can preconceived people on the whole poster design an impression, to attract public attention.

The graphics are the most concise expression of the language is easy to understand the artistic expression. Poster design success and integration of graphical elements have a great relationship. Graphics can be understood as all except photography and shape of FIG. Graphic its unique phenomena force, in Layout constitution show a unique visual appeal. Graphics are important to the formation of creative advertising character and improve visual attention in the plane of the constituent elements. Fun integrate graphic elements can be positioned about poster design an overall spread of ideas. Poster design into the fun is the most intuitive graphical elements can cause people's attention, stimulate interest in exploring elements. Fun graphic elements more advantages than the text and color. The size of the area occupied by the graphics often determines the intensity of the propagation of advertising posters. Graphics large area can heighten the atmosphere, produce a strong visual impact. Especially large-scale outdoor advertising poster design, but also need to grab the audience's attention with vivid graphics, to instantly convey information purposes. Into a large number of interesting graphic elements can cause everyone's attention, theme conducive poster design outstanding, conducive to the implementation-related concept of cultural presentation of change related to the concept of promoting change related concept of culture, promote the transformation of the cultural field of important factor.

\section{The Role of Poster Design Element Fun Graphic Applications}

Performance poster design work is a product design start, referred to convey the contents of liquidity practices, poster design, accuracy, creativity, simplicity, is a measure of poster design standards, but also the poster designer should poster design follow design thinking and expression. Various promotional poster design concept, poster designer for all is an important challenge to be able to make their own design products are recognized by everyone, it is the source of poster designer rewarding.

With the development of society, not only as graphical elements constitute one element of poster design, it should also have a wide range of interesting, poster design in order to improve taste. Graphic Communication Arts is a people of important media, full of graphics can pull the entire conceptual design interesting, to some extent, profundity. Conducive to the promotion poster design concepts to give public recognition. 
Interesting graphic elements into poster design to increase the expression of creative graphic language. Applications to some extent interesting graphic elements on the poster design can distinguish between normal, resulting in the above visual impact. Graphic design is the poster carrier, carrying large amount of information, you need to approach different manifestations, fun graphics it is one of them, but it is also the most important of which can cause the audience as a means of visual impact visually.

With the form of development of the times, the information carrier performance varied, fun poster design graphic elements is an important element of the application, able to give the audience a profound impact on the projected information and deepen the potential impact on the entire poster design. Graphic manipulation participation into the following six, this is the most common way to use interesting graphics.

(1) three-dimensional space can not be contradictory to complete three-dimensional shape, flat in the show, highlighting the unique concept out, show diversification related concepts in two-dimensional space.

(2) the use of common type, edge-collinear, coplanar symbiotic way to produce a third form, in favor of public acceptance.

(3) the variability in the natural form of graphics, seek rationality visual unreasonable, avoiding sharp visual conflict.

(4) the substance in two different spatial restructuring, constitutes a shared space Graphics

(5) the use of the principle of light projection method, find a different variation of the shape of light and shadow

(6) graphic text, graphic text of

(7) by the gradient of an object to another object shape

Seven of expression is above poster design fun graphic elements of applications, the most common way to promote one of the important factors in the use of the above transformation techniques of application of these techniques to a certain extent, can be seen above the important cultural one change mode, you can see one of the important concept of cultural revolution above centuries.

Interesting graphic elements into poster design to increase the accuracy of the graphic language. Dissemination of information by the realities of technical limitations and related laws and regulations, relatively speaking, the dissemination of information is free, but in the formal poster ads need clear language of art to show the authenticity of an existing phenomenon, blurred culture concept conceptual design can not meet everyone's needs, but also displayed an important reason for the concept of surface phenomena transition above changes.

Graphical representation of the text relative to the subtle, there are straightforward representation of the effect, people can intuitively understand the concept of positive poster design design want to express, but different people understanding is not the same, this and causing the same graph, there are multiple layers of meaning.

Who is a regular poster design receptors, and the recognition of a complex, changing organism, has a cold fuzzy thinking, difficult to use language to express accurate information, plus the reduction in the different social background from this way the performance of the graphics, it is possible to some extent be able to disseminate relevant information to facilitate the dissemination.

Fun graphic elements into poster design increases the simplicity of the graphic language. Poster design creative graphics can be shown, the graphics are dressing external image, important information and media image among the audience. Media presentation more intuitive and more capable of rendering the overall effect of the poster design to promote the dissemination of information, the faster, the better the results.

Poster Design fun graphics are a visual symbol, the human visual image is projected by the external things to the retina in the nerve transferred to the identification, then respond by the brain. External image more clear, more direct response of the brain. Judging from the shape of the object, the less hierarchical objects, visual recognition faster. From the point of view of plane figures and simple graphics than the regular complex irregular pattern recognition faster, which stressed the 
importance of poster design element fun graphic simplicity of application.

Fun graphic elements into poster design can be more close to the people. Poster design in the face of the main audience is the normal person, so when the main design must be practical. Fun graphics into the poster designs, interesting graphic elements popularity, limit break area.

Poster Design graphic elements into the fun is to promote the overall design concept can be known by the public, this is the ultimate purpose of the design. Economic development level is not the same, not the same cultural acceptance, political atmosphere is not the same, so when the poster design should fully take into account various factors, and promote the popularization of acceptance of poster design.

\section{References}

[1] Huang Huang. graphical image of the city poster language research [D]. Guangxi Normal University. 2011.

[2] Guo Hongxiu. On three elements poster design [J]. Modern decor (theory). 2011, (11).

[3] Bai Lu. Poster in the graphic language of analysis [J]. Writer. 2011, (18).

[4] Qin Fangfang. On the domestic modern poster creative ideas and thinking [J]. Creativity and Design. 2011, (03).

[5] Zhou Rong, Luo Hui, Wang Hongliang. Japan Graphic designer Takashi Akiyama Interview [J]. Decoration, 2005, (3): 38-39.

[6] Lin Shuangqing, Zhang Jinping. Images of Graphic Language in Packaging Design [J]. Radio and TV University. 2012, (02).

[7] Zhu Beibei. use. On the graphic design aesthetic consciousness [J]. Xiang Chao (second half) 2011, (12).

[8] Sun Dandan. On the poster layout constitute [J]. The Youth Writers. 2011, (19).

[9] Jiao Zhentao. Chinese traditional graphic design study [J]. Decoration. 2003, (10).

[10] Dong Yueyun. Chinese traditional cultural elements in your poster in the use of [J]. Popular Literature (theory), 2009, (02). 